\title{
Le bibliotechnicien et le technicien en documentation : douze ans plus tard
}

The library technician and the documentation technician: twelve years later

\section{El bibliotécnico y el técnico de la documentación: doce años más tarde}

Denis Plante, Michelle Ouellette et Lise Lachance

Volume 26, numéro 3, septembre 1980

URI : https://id.erudit.org/iderudit/1054228ar

DOI : https://doi.org/10.7202/1054228ar

Aller au sommaire du numéro

Éditeur(s)

Association pour l'avancement des sciences et des techniques de la documentation (ASTED)

\section{ISSN}

0315-2340 (imprimé)

2291-8949 (numérique)

Découvrir la revue

\section{Citer cet article}

Plante, D., Ouellette, M. \& Lachance, L. (1980). Le bibliotechnicien et le technicien en documentation : douze ans plus tard. Documentation et bibliothèques, 26(3), 151-159. https://doi.org/10.7202/1054228ar
Résumé de l'article

Les bibliotechniciens et les techniciens de la documentation constituent un groupe important du monde documentaire québécois. À partir de deux enquêtes, l'une en 1975, l'autre en 1980, cet article vise à fournir des données quantitatives sur ces techniciens dans le domaine de l'emploi et des tâches occupées et à rendre compte de leur perception de leur carrière à long terme.
Tous droits réservés (C) Association pour l'avancement des sciences et des techniques de la documentation (ASTED), 1980
Ce document est protégé par la loi sur le droit d'auteur. L'utilisation des services d'Érudit (y compris la reproduction) est assujettie à sa politique d'utilisation que vous pouvez consulter en ligne.

https://apropos.erudit.org/fr/usagers/politique-dutilisation/ 


\title{
Le bibliotechnicien et le technicien en documentation: douze ans plus tard*
}

\author{
Denis Plante \\ Service des archives \\ Université de Montréal \\ Michelle Ouellette \\ Bibliothèque municipale de Montréal \\ Lise Lachance \\ Chambre des notaires \\ Montréal
}

Les bibliotechniciens et les techniciens de la documentation constituent un groupe important du monde documentaire québécois. À partir de deux enquêtes, l'une en 1975, l'autre en 1980, cet article vise à fournir des données quantitatives sur ces techniciens dans le domaine de l'emploi et des tâches occupées et à rendre compte de leur perception de leur carrière à long terme.

The library technician and the documentation technician: twelve years later

Library technicians and documentation technicians form an important group in the Quebec world of documentation. Based on the results of two surveys conducted in 1975 and 1980, this article is intended to provide quantitative data on technicians in the employment ranks and positions held. It also describes the technicians own perceptions of their careers.

\section{El bibliotécnico y el técnico de la documentación: doce añosimás tarde}

Los bibliotécnicos y los técnicos de la documentación constituyen un grupo importante en el mundo de la documentación quebecense. Utilizando los resultados de dos investigaciones, una hecha en 1975, otra en 1980, el autor intenta dar datos cuantitativos sobre estos técnicos en cuanto al empleo y a las posiciones ocupadas; explica también la percepción que tienen los técnicos de su carrera en el futuro.

Le développement de la technologie a entraîné des changements majeurs dans un grand nombre de métiers et professions. Souvent, ces transformations ont à leur tour amené des innovations, parmi lesquelles l'apparition d'une autre catégorie d'employés spécialisés: le technicien. Au Québec, il y a maintenant douze ans que les premiers diplômés en bibliotechnique ont commencé à s'intégrer au personnel qualifié du domaine de la documentation.

\footnotetext{
* Les auteurs remercient monsieur Alain Rigollot, informaticien à l'Université de Montréal, et madame Monique Lefebvre Poulin, bibliotechnicienne, pour leur précieuse collaboration.
}

Les premières années ont été utilisées à situer les bibliothécaires et les bibliotechniciens les uns par rapport aux autres. On a procédé par "essais et erreurs", faisant les indispensables ajustements de part et d'autre. II y a loin de la théorie à la pratique: les techniciens étaient-ils vraiment capables d'accomplir toutes les tâches pour lesquelles on les disait formés? Qu'allaient devenir les bibliothécaires, déchargés de certaines tâches précé. demment accomplies par eux et maintenant confiées aux techniciens?

Peu à peu, les fonctions se sont défi- 
nies et les techniciens ont fait leurs preuves. En 1975, le Comité des bibliotechniciens de l'ASTED décida de mener une enquête pour tenter de faire le point sur leur situation. Les résultats compilés de cette enquête prirent la forme d'une étude, parue en mai 1975.

Depuis lors, le cours de bibliotechnique s'est transformé en celui de techniques de la documentation. L'arrivée des diplômés ayant une maîtrise en bibliothéconomie a contribué à modifier, à son tour, la fonction du bibliothécaire. La situation économique générale s'est détériorée, entraînant des coupures de budget et de postes un peu partout. Les A.A.C.R., les I.S.B.D., l'informatique, l'indexation, le catalogage avant publication, la gestion de documents sont devenus des réalités vécues quotidiennement par bon nombre.

\section{Comment et en quoi cela a-t-il affecté le technicien?}

En février 1980, un questionnaire comparable en plusieurs points à celui utilisé en 1975 était adressé aux techniciens répertoriés dans l'annuaire ${ }^{1}$. En 1975, alors que le nombre estimé de diplômés était de 1200 , il y avait eu 415 répondants. À I'hiver 1980 , le nombre de diplômés se situait aux environs de 1800 . De ce nombre, seulement 347 ont répondu à l'enquête.

Quelles que soient les raisons de cette baisse importante, il est difficile de prétendre brosser un tableau d'ensemble très exact avec moins de $20 \%$ de répondants. En dépit de cette réserve, les résultats obtenus méritent d'être connus car il s'en dégage une logique renforcée par les comparaisons avec certaines des données les plus significatives de l'enquête de 1975.

\section{Profil du technicien}

En 1968, on obtenait son diplôme de technicien à l'âge de 21 ou 22 ans environ. Avec les changements dans le système scolaire, on sort aujourd'hui du cégep, diplôme en poche, à 19 ou 20 ans. En re-

1. Cet annuaire est constitué à partir de la liste des finissants en bibliotechnique et en techniques de la documentation. vanche, à cause du contingentement, on en sort en rangs moins serrés qu'il y a quelques années. Toutes ces raisons expliquent sans doute qu'en 1980, l'âge moyen du technicien soit de 28 ans. Quant à la majorité féminine de la profession, elle s'affiche une fois de plus avec $86,5 \%$ de répondants féminins et $13,5 \%$ de répondants masculins. Dans une proportion de $84,4 \%$, le technicien a obtenu son diplôme à la suite des trois ans de cours réguliers prévus pour sa formation. Ajoutons que $35,8 \%$ des répondants ont entre un et quatre ans d'expérience et que $64,1 \%$ ont de cinq à douze ans d'expérience.

\section{Situation de l'emploi}

Les premières années de l'apparition du bibliotechnicien dans le domaine de la documentation ont la double particularité d'avoir été à la fois une époque pionnière et une sorte d'âge d'or de l'emploi. Si le technicien était parfois accueilli avec certaines réticences et rencontrait une certaine méfiance, par contre il n'avait pas à craindre le chômage. En quelques années, la situation s'est totalement modifiée: bien intégré, le technicien s'est retrouvé victime d'une saturation du marché du travail plus rapide que prévue.

Les solutions trouvées à ce dernier problème ont été, d'une part, le contingentement des candidatures acceptées pour recevoir la formation de technicien et, d'autre part, la transformation du cours de bibliotechnique en cours de techniques de la documentation. Le cours remanié avait pour but de s'adapter à l'évolution rapide du domaine et surtout d'ouvrir de nouveaux champs d'emploi, dont les archives et les librairies.

II y a maintenant cinq ans que s'exerce le contingentement et deux ans que les techniciens en documentation ont pris place à côté des bibliotechniciens sur le marché du travail. L'enquête 1980 a tenté de cerner l'efficacité des mesures prises par leur répercussion sur la situation de l'emploi.

Disons tout de suite que la formulation du questionnaire a laissé échapper une donnée précieuse, soit le nombre de chô- 
meurs par défaut d'emploi. En effet, un certain nombre de répondants se disaient sans emploi, mais souvent sans préciser s'il s'agissait d'un choix volontaire. Dans une profession à majorité féminine où nombre de jeunes femmes interrompent temporairement ou définitivement leur carrière à l'occasion d'une grossesse, l'absence de cette précision rend la donnée inutilisable.

Par contre, les résultats obtenus sur d'autres points permettent des recoupements très intéressants. Ainsi, même si $90 \%$ des répondants occupent ou ont occupé un emploi à temps plein, $25 \%$ occupent ou ont occupé un emploi à temps partiel et $26 \%$ occupent ou ont occupé un emploi occasionnel, dit aussi à contrat. Dans chacun de ces types d'emploi, temps plein, temps partiel, occasionnel, le nombre de postes occupés a varié entre un et trois.

En d'autres mots, même si la quasitotalité des techniciens réussit à obtenir un emploi à temps plein, une proportion importante doit avoir recours aux emplois à temps partiel ou occasionnels. Le nombre moyen de postes occupés indique nettement une situation du marché du travail assez difficile. Par exemple, il est significatif qu'aucun des répondants, même parmi ceux ayant douze ans d'expérience, n'ait occupé plus de trois postes à temps plein. De plus, lorsque le nombre de postes occupés à temps partiel ou à contrat s'élève jusqu'à trois par type et par répondant, la conclusion s'impose d'elle-même.

La situation, d'ailleurs, se dessine de façon encore plus marquée si on se penche sur la répartition de l'emploi selon l'année de promotion (voir Tableau 1).

En comparant l'année 1971, la première au cours de laquelle les diplômés n'ont pas tous obtenu un poste à temps plein, avec l'année 1979, on constate qu'en 1979 il y a eu $18 \%$ d'emplois à temps plein en moins, mais $8 \%$ d'emplois à temps partiel et $28,8 \%$ à contrat en plus. En gardant en mémoire le contingentement qui a certainement diminué le nombre des finissants de 1979 par rapport à ceux de 1977, le tableau n'est guère réjouissant.

Au niveau des secteurs de travail (voir Tableau 2), les résultats des deux enquêtes restent assez conséquents, puisqu'on enregistre des hausses appréciables, mais sans démesure, dans l'entreprise privée et le secteur municipal. Par contre, la baisse spectaculaire dans le secteur para-public, qui inclut les écoles, les cégeps et les universités, reste très sujette à caution. II est fort douteux, même en supposant qu'il n'y ait pas eu de progression dans ce secteur, que les emplois déjà existants aient été abolis ou laissés vacants en assez grand nombre pour justifier une baisse de près de la moitié du résultat obtenu en 1975.

\section{Tableau 1:}

\section{Situation de l'emploi}

Répartition de l'emploi entre 1968-1979 (les répondants n'ont pas nécessairement occupé ces emplois l'année même de l'obtention de leur D.E.C.)

\begin{tabular}{|c|c|c|}
\hline $\begin{array}{l}\text { TEMPS } \\
\text { PLEIN }\end{array}$ & $\begin{array}{l}\text { TEMPS } \\
\text { PARTIEL }\end{array}$ & $\begin{array}{l}\text { OCCASIONNEL } \\
(\dot{A} \text { CONTRAT })\end{array}$ \\
\hline $\begin{array}{lr}\text { 1968: } & 100 \% \\
\text { 1969: } & 100 \% \\
\text { 1970: } & 100 \% \\
\text { 1971: } & 95 \% \\
\text { 1972: } & 100 \% \\
\text { 1973: } & 94,1 \% \\
\text { 1974: } & 96,1 \% \\
\text { 1975: } & 93,7 \% \\
\text { 1976: } & 83,7 \% \\
\text { 1977: } & 93,5 \% \\
1978: & 86 \% \\
1979: & 77,7 \%\end{array}$ & $\begin{array}{c}- \\
25,5 \% \\
- \\
20 \% \\
25,5 \% \\
29,4 \% \\
15,3 \% \\
21,1 \% \\
23,2 \% \\
35,4 \% \\
43,4 \% \\
38,8 \%\end{array}$ & $\begin{array}{r}- \\
- \\
14,2 \% \\
10 \% \\
23,2 \% \\
23,5 \% \\
36,8 \% \\
22,9 \% \\
41,8 \% \\
35,4 \% \\
30,4 \% \\
38,8 \%\end{array}$ \\
\hline
\end{tabular}

Tableau 2:

\section{Emplois par secteur de travail}

\begin{tabular}{|l|r|r|}
\hline & \multicolumn{1}{|c|}{1980} & \multicolumn{1}{|c|}{1975} \\
\cline { 2 - 3 } Entreprise privée & $9,2 \%$ & $5,8 \%$ \\
Gouvernement & $28,2 \%$ & $25 \%$ \\
Para-public & $35,4 \%$ & $65,1 \%$ \\
Municipal & $9,2 \%$ & $4,5 \%$ \\
Autres & $9,2 \%$. & \\
Sans réponse * $^{*}$ & $8,6 \%$ & \\
\hline
\end{tabular}

*Seul chiffre disponible sur les sans emplois.

Quand on passe aux genres de bibliothèque (voir Tableau 3), les mêmes divergences se manifestent, particulièrement marquées dans les bibliothèques gouver- 
nementales, ainsi que dans les bibliothèques scolaires. Dans le cas des bibliothèques gouvernementales, l'enquête de 1975 les regroupait sous la rubrique Spécialisée. Fonction publique. II est possible qu'un certain nombre de répondants à l'enquête 1980 se soient enregistrés à la catégorie Bibliothèques spécialisées plutôt qu'à Bibliothèques gouvernementales. Toutefois, rien ne permet de l'affirmer avec certitude. Dans le cas des bibliothèques scolaires, il faut mettre en doute soit les résultats de l'enquête, soit la tendance pourtant assez généralisée consistant à embaucher des bibliotechniciens comme responsables des bibliothèques d'école.

\section{Tableau 3:}

\section{Emplois par genre de bibliothèque}

\begin{tabular}{|l|r|r|}
\hline & 1980 & 1975 \\
\cline { 2 - 3 } Gouvernementale & $11,5 \%$ & \\
Universitaire & $11,2 \%$ & $13,7 \%$ \\
De collège & $7,7 \%$ & $20,5 \%$ \\
Scolaire/élémentaire & $5,4 \%$ & $30,1 \%$ \\
Scolaire/secondaire & $16,7 \%$ & \\
Spécialisée & $18,4 \%$ & \\
Publique & $9,7 \%$ & $5 \%$ \\
Librairie & $0,5 \%$ & \\
Dépôt d'archives & $4,9 \%$ & \\
Autres & $5,8 \%$ & \\
Sans réponse * & $8,6 \%$ & \\
Fonction publique & & \\
spécialisée & & $31,1 \%$ \\
\hline
\end{tabular}

*Seul chiffre disponible sur les sans emplois.
Par contre, le $0,5 \%$ enregistré dans le domaine de la librairie semble conforme à ce qu'on sait de ce milieu de travail qui est resté quasi totalement fermé au technicien, en dépit du cours de techniques de la documentation qui voulait permettre au technicien d'y trouver place.

Enfin, mentionnons l'agréable surprise de la percée du technicien dans le domaine des archives et de la gestion de documents. Malgré les pronostics de l'enquête Reicher, 12 techniciens jusqu'en 1980, ce domaine a accueilli le technicien très favorablement et on peut espérer que ce débouché prendra encore de l'importance d'ici quelques années.

\section{Description des tâches}

Au sujet de la fonction, on enregistre des changements dans les champs classiques d'activité du technicien: ainsi, en 1975 $56,1 \%$ des répondants travaillaient au moins en partie dans les services techniques. En 1980, 74,6\% déclarent le faire. De même, en 1975, 40,3\% seulement des techniciens étaient employés aux services publics. En 1980, la proportion atteint $66,9 \%$. Parmi les techniciens qui travaillent au moins en partie dans les services publics, près de la moitié $(47.5 \%)$ fait de la référence.

Tableau 4: Description des tâches (1980)

\begin{tabular}{|l|c|c|c|c|c|c|c|}
\hline \multicolumn{1}{|c|}{ TÂCHES } & Universitaires & De collège & Scolaires & Publiques & Spécialisées* & TOTAL & \\
\hline Acquisitions & 12 & 7 & 64 & 17 & 53 & 153 & $44 \%$ \\
\hline Catalogage & 24 & 12 & 57 & 23 & 72 & 188 & $54,1 \%$ \\
\hline Classification & 7 & 10 & 52 & 21 & 57 & 147 & $42,3 \%$ \\
\hline Indexation & 1 & 2 & 29 & 9 & 53 & 97 & $27,9 \%$ \\
\hline Référence & 8 & 7 & 61 & 22 & 67 & 165 & $47,5 \%$ \\
\hline Prêt & 8 & 5 & 65 & 18 & 63 & 168 & $48,4 \%$ \\
\hline Audiovidéothèque & 3 & 6 & 31 & 6 & 19 & 65 & $18,7 \%$ \\
\hline Périodiques & 4 & 12 & 54 & 13 & 58 & 138 & $37,7 \%$ \\
\hline $\begin{array}{l}\text { Publications } \\
\text { officielles }\end{array}$ & 2 & 4 & 23 & 4 & 37 & 70 & $20,1 \%$ \\
\hline
\end{tabular}

*Incluant les centres de documentation. 
II serait intéressant d'analyser en détail les endroits où se manifestent le plus ces changements, en comparant les résultats de 1975 avec ceux de 1980 quant à la répartition des fonctions selon les genres de bibliothèque. Toutefois, seulement 317 réponses en 1980 contre 415 en 1975 ont été obtenues aux questions sur le sujet et la répartition entre les divers genres de bibliothèques est sensiblement différente de celle relevée en 1975 (voir Tableaux 4 et 5).

Seul le secteur des bibliothèques publiques permet d'établir certains recoupements, puisque $5 \%$ des répondants de 1975, soit une vingtaine de personnes, provenaient de ce milieu, alors que le 9,2\% de 1980 totalise environ trente personnes. Or, on y retrouve une augmentation sensible du nombre de techniciens dans toutes les tâches, sauf les publications officielles.

En 1975, toute une série de tâches (archives, gestion de documents, etc.) n'existait pas ou n'était pas confiée au technicien. De ce côté, les résultats de l'enquête indiquent que la formation en techniques de la documentation semble un succès puisque $17 \%$ des répondants accomplissent une ou plusieurs de ces nouvelles tâches. Encore une fois, insistons sur les archives et la gestion documentaire, puisque dix-sept personnes ont indiqué travailler dans le domaine des archives et que vingt-trois autres ont signalé dans leur description de poste des tâches reliées à ce domaine.

Par rapport à 1975, on peut avancer I'hypothèse que les tâches confiées au technicien correspondent de plus en plus à sa formation et exploitent de mieux en mieux son potentiel. Pourtant, alors qu'en $197581 \%$ des répondants estimaient accomplir un travail de technicien, seulement $76,6 \%$ d'entre eux répondent en ce sens en 1980 .

\section{Niveau de responsabilité}

II y a quelqyes années, le reproche le plus fréquent des techniciens face à leur métier était la sous-exploitation de leur potentiel, ce qui impliquait le désir d'accéder à des postes de responsabilité.

Pourtant, en 1975 comme maintenant, on retrouvait des bibliotechniciens en charge de personnel, de services, voire même de bibliothèques (voir Tableaux 6 , 7 et 8).

Or, la comparaison des deux enquêtes permet de constater que la situation ne s'est guère modifiée, sinon pour accuser une forte diminution dans la responsabilité de service. Du côté des archives et de la gestion de documents, il convient de re-

Tableau 5: Description des tâches (1975)

\begin{tabular}{|l|c|c|c|c|c|c|c|}
\hline TÂCHES & Universitaires & De collège & Scolaires & Publiques & Spécialisées & TOTAL & \\
\hline Acquisitions & 14 & 21 & 96 & 8 & 59 & 198 & $47,7 \%$ \\
\hline Catalogage & 18 & 48 & 105 & 13 & 94 & 278 & $67 \%$ \\
\hline Classification & 8 & 34 & 99 & 13 & 69 & 223 & $53,7 \%$ \\
\hline Référence & 19 & 26 & 88 & 12 & 72 & 217 & $52,3 \%$ \\
\hline Prêt & 21 & 26 & 90 & 11 & 69 & 217 & $52,3 \%$ \\
\hline Audiovidéothèque & 3 & 17 & 55 & 1 & 30 & 106 & $25,5 \%$ \\
\hline Périodiques & 9 & 30 & 85 & 12 & 64 & 200 & $48,2 \%$ \\
\hline $\begin{array}{l}\text { Publications } \\
\text { officielles }\end{array}$ & 4 & 9 & 36 & 5 & 43 & 97 & $23,4 \%$ \\
\hline Autres & 5 & 8 & 8 & 2 & 24 & 47 & $11,3 \%$ \\
\hline
\end{tabular}


marquer le nombre phénoménal de seize personnes responsables, sur les dix-sept travaillant dans ce nouveau secteur.

Fait à mentionner, $55 \%$ des femmes et $52,5 \%$ des hommes occupent des postes de responsabilité. De ce côté, on peut parler d'égalité des chances dans l'emploi.

\section{Plan de carrière}

En tenant compte des chiffres mentionnés plus haut, il est évident que le technicien a progressé dans sa profession. Cependant, l'avenir s'annonce sous d'autres couleurs, si on considère qu'à la question portant sur les possibilités d'avancement, quelque $60,5 \%$ des répondants n'en voient pas dans leur service actuel et $56,1 \%$ n'en voient même pas dans leur secteur de travail (voir Tableau 9).

À dire vrai, les préoccupations immédiates du technicien portent surtout sur l'obtention d'un poste permanent; cette préoccupation vient en tête de liste des raisons ayant motivé un changement d'emploi. Encore une fois, soulignons les très

\section{Tableau 6:}

Niveau de responsabilité

\begin{tabular}{|l|r|c|}
\hline En charge de: & 1980 & 1975 \\
\cline { 2 - 3 } Bibliothèque & $21,3 \%$ & $26,3 \%$ \\
Dépôt d'archives et & & \\
gestion des documents & $4,6 \%$ & - \\
Librairie & $0,2 \%$ & - \\
Service & $16,1 \%$ & $40 \%$ \\
Personnel & $11,2 \%$ & $14 \%$ \\
Autre(s) & $6,6 \%$ & - \\
\hline
\end{tabular}

nombreux postes occasionnels, dits aussi à contrat, dont doivent de plus en plus souvent se contenter les techniciens, parfois pour de longues périodes. Bien que préférables au chômage, ils contribuent quand même à la précarité du plan de carrière ouvert au technicien.

En dépit de la situation de l'emploi, le problème le plus crucial du technicien réside dans la question du plan de carrière. Formé dans le but d'exécuter des tâches techniques et non pas en vue d'exercer des responsabilités, le technicien y accède cependant grâce à son potentiel individuel. Actuellement, c'est l'obtention de responsabilités qui tient lieu de plan de carrière

Tableau 7: Répartition du niveau de responsabilité (1980)

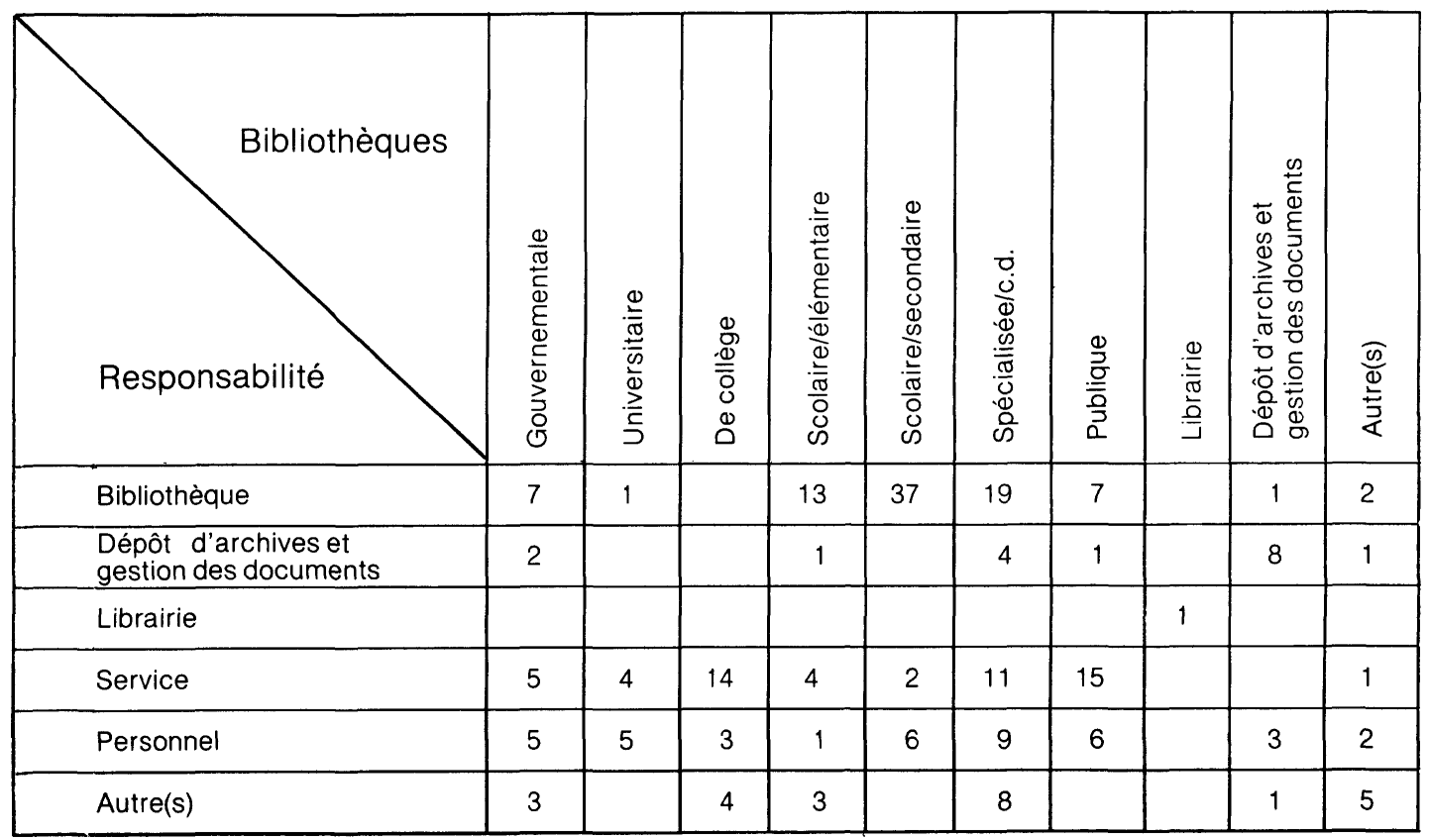


Tableau 8: Répartition du niveau de responsabilité (1975)

\begin{tabular}{|c|c|c|c|c|c|}
\hline \multicolumn{6}{|l|}{ Bibliothèques } \\
\hline Responsabilité & Universitaire & De collège & Scolaire & Spécialisée & Municipale \\
\hline Bibliothèque & 3 & 6 & 70 & 25 & 5 \\
\hline Service & 14 & 46 & 47 & 47 & 13 \\
\hline Personnel & 6 & 14 & 25 & 9 & 4 \\
\hline
\end{tabular}

\section{Tableau 9: Plan de carrière}

\begin{tabular}{|c|c|c|c|}
\hline \multicolumn{4}{|c|}{ Possibilités d'avancement dans: } \\
\hline & & 1980 & 1975 \\
\hline Votre service actuel: & OUI & $8,9 \%$ & $21,5 \%$ \\
\hline Votre service actuel: & NON & $60,5 \%$ & $69 \%$ \\
\hline Votre secteur actuel: & OUI & $12,4 \%$ & $32 \%$ \\
\hline Votre secteur actuel: & NON & $56,1 \%$ & $52 \%$ \\
\hline
\end{tabular}

\section{Tableau 10: Plan de carrière}

Lorsque vous avez changé d'emploi, s'il y a lieu, ces changements étaient dus à quelle(s) raison(s):

\begin{tabular}{|lc|}
\hline Pour un emploi permanent & $22,7 \%$ \\
Pour un emploi plus rémunérateur & $10,6 \%$ \\
Insatisfaction de l'emploi précédent & $13,9 \%$ \\
Pour un ensemble de tâches qui correspondent mieux & \\
à vos besoins ou capacités & $12,4 \%$ \\
Pour une responsabilité de service & $5,7 \%$ \\
Pour une responsabilité de secteur & $0,9 \%$ \\
Pour un changement de type de bibliothèque & $5,1 \%$ \\
Changement de localité & $10 \%$ \\
Autres & $18,4 \%$ \\
\hline
\end{tabular}

au technicien. Hors, malgré (ou peut-être à cause) des responsabilités confiées à un grand nombre de techniciens, les possibilités d'avancement sont estimées à $8,9 \%$ dans le service et à $12,4 \%$ dans le secteur. Cette perception de la situation a des conséquences directes sur les raisons incitant un technicien à changer d'emploi. Mis à part l'attrait du poste permanent, le technicien recherchera surtout un poste corres. pondant mieux à ses besoins et capacités, ce qui implique en général une certaine insatisfaction quant au poste qu'il désire quitter. Bien entendu, une rémunération supérieure entrera, elle aussi, en ligne de compte (voir Tableau 10).

Placidité ou réalisme? $43,9 \%$ comptent conserver leur emploi, mais $35 \%$ en chercheront un nouveau, à plus ou moins long terme. Même si $66,9 \%$ des répondants disent vouloir rester dans le domaine, $11,8 \%$ comptent le quitter définitivement et $18,5 \%$ désirent retourner aux études dans un autre champ de connaissance. Enfin, 8,5\% seulement envisagent la maîtrise en biblio- 
théconomie, seule solution de continuité possible, présentement, dans la carrière du technicien. Étant donné que, jusqu'à maintenant, seulement trois techniciens ont opté pour la bibliothéconomie et complété une maîtrise, cette faible proportion semble d'ailleurs dépasser la réalité.

Faut-il conclure que le technicien, dans l'ensemble, est résigné à la situation? Chose certaine, il y cherche des palliatifs. Un autre sondage effectué voici deux ans auprès de 253 techniciens démontrait que $50 \%$ d'entre eux avaient suivi d'autres cours depuis l'obtention de leurs diplômes. $92 \%$ se disaient intéressés à se perfectionner en sciences de la documentation, dont $41 \%$ en vue de l'obtention d'un baccalauréat spécialisé.

Depuis la transformation du baccalauréat en maîtrise en bibliothéconomie, plusieurs estiment, tant parmi les techniciens que les bibliothécaires, qu'il manque un échelon naturel parmi le personnel spécialisé en services documentaires. Un groupe de travail de l'ASTED a élaboré, à partir de cette hypothèse, un programme de premier cycle visant comme clientèle des techniciens diplômés. Le projet vise à solutionner l'absence de plan de carrière à long terme pour le technicien.

Il y a une quinzaine d'années, c'était autour du bien-fondé d'un cours de bibliotechnique que s'alimentait le débat. L'apparition du technicien a réglé une bonne part des problèmes de personnel qualifié que connaissait le domaine de la documentation. Maintenant, le problème a changé de niveau et d'aspect. Dire que les techniciens aspirant à de plus hautes fonctions ont été "mal orientés" et qu'ils auraient dû dès le départ opter pour la bibliothéconomie, ou soutenir qu'ils n'ont qu'à le faire maintenant, est par trop simpliste et tout à fait irréaliste.

L'absence de motivation du technicien après quelques années de travail et le changement d'orientation de certains parmi les plus dynamiques ont tous deux pour cause première le rapide plafonnement professionnel auquel est confronté le technicien. C'est d'abord la prise de conscience de tous les aspects du problème qui aidera à trouver une solution, que celle-ci réside ou non dans le projet de baccalau- réat. D'ici à ce qu'on aboutisse à une décision sur le sujet, la création de postes de chef d'équipe pourrait être un premier pas important à franchir.

\section{Conclusion}

La transformation du cours de bibliotechnique en techniques de la documentation a assuré une heureuse percée, de la part du technicien, du côté des archives et de la gestion des documents, domaines qui s'annoncent comme des débouchés très intéressants pour les prochaines années. Il en va de même dans le secteur privé, qui se révèle de plus en plus un bon milieu potentiel; on pourrait, selon les données de notre enquête, dire la même chose du secteur municipal. Ces nouvelles possibilités d'emploi aideront sans doute à régler le problème chronique des emplois occasionnels, ainsi que son corollaire, le chômage.

Du côté des postes de responsabilité, il faut voir les deux côtés de la médaille. D'une part, ils constituent un stimulant actif pour le technicien désireux d'exploiter toutes ses capacités. D'autre part, ils ne sont qu'un palliatif à l'absence d'un plan de carrière réel. De plus, ils servent parfois de prétexte à l'accomplissement de tâches de niveau professionnel par un technicien. Cet aspect de travail à rabais ne peut que nuire au technicien.

Les faibles possibilités d'avancement dans les secteurs de travail et les services doivent être solutionnées. Le problème devient de plus en plus pressant au fur et à mesure que s'élèvent l'âge et les années d'expérience du technicien. À tout le moins, la création de postes de chef d'équipe devrait se faire le plus rapidement possible.

Enfin, même si le but poursuivi est encore lointain, le projet d'un premier cycle universitaire en sciences de la documentation mérite d'être envisagé. En permettant au technicien de modifier son statut, il lui assurerait également un plan de carrière à plus longue échéance. Cela retiendrait peui-être dans le milieu certains des éléments qui, faute d'avenir intéressant, changent maintenant d'orientation. 
En douze ans à peine, le technicien s'est taillé une place parmi le personnel du monde de la documentation. Il a évolué en souplesse, tout en stabilisant sa position dans des fonctions convenant à sa formation. On constate aujourd'hui une spécialisation par poste, alors qu'auparavant il accomplissait un peu de toutes les tâches techniques. II a également réussi à obtenir des postes de responsabilité dans des proportions telles qu'on se doit de souligner ses capacités. En terminant, disons que si le technicien a mené son combat souvent de manière isolée, le petit nombre d'entre eux actifs au sein de leur association professionnelle a sûrement contribué plus qu'il n'y paraît à faire reconnaître son professionalisme.

\title{
La Belle Peliure de Montréal Inc.
}

\author{
RELIURE DE TOUS GENRES \\ BIBLIOTHEQUE - PERIODIQUES \\ RELIURE PLASTIFIEEE \\ RELIURE COMMERCIALE \& D'ÉDITION \\ RELIURE DE LUXE
}

8377, BOUL. LANGELIER

MONTREAL, QUEBEC, H1P 2C3

(514) $324-2610$

Membre de l'A.R.B.Q. 